\title{
Licia Ríos
}

Becaria de Posgrado Tipo II CONICET con sede en el CIUyT (Centro de Investigaciones Urbanas y Territoriales), Facultad de Arquitectura y Urbanismo, Universidad Nacional de La Plata. Maestranda en Ciencias del Territorio y doctoranda en Arquitectura y Urbanismo, FAU-UNLP. Auxiliar de Curso Diplomado en Cátedra de Planeamiento Físico No 1. FAU-UNLP. Correo electrónico:licia.rios@hotmail.com 


\section{$\underline{\text { Resumen }}$}

Como tesis del ensayo se plantea que tanto en México, como en Argentina, en el proceso de las políticas de ordenamiento territorial municipal, se visualizan procesos estructurales que dificultan su concreción, desvalorizándolas como políticas transversales al conjunto de acciones que tienen por objetivo el desarrollo de los ámbitos locales.

Para demostrar esta tesis se revisan en primer lugar los aspectos conceptuales fundamentales para el entendimiento del nuevo rol del ordenamiento territorial en el contexto municipal. Luego se indagan aspectos clave de la etapa de formulación de los principales instrumentos de la política de ordenamiento en dos municipios de ambos países: Tequisquiapan (estado de Querétaro) y Chascomús (provincia de Buenos Aires). Finalmente se presentan las conclusiones preliminares, buscando entender los obstáculos encontrados y encontrar algunos indicios de cambio en la forma de hacer las políticas.

\section{Palabras clave}

Políticas Municipales, Ordenamiento Territorial.

\section{Abstract}

The hypothesis of this essay argues that in Mexico, and Argentina, in the process of development of municipal land management policies structural processes are displayed that impede their realization, devaluing them as transversal policies to the set of actions directed to the development of local areas.

To prove this hypothesis the paper first reviews the concepts on which the new role of land use in the municipal context is based. It then probes the main tools developed within the framework of the management policy in two municipalities of both countries: Tequisquiapan (Edo. of Queretaro) and Chascomús (Provincia de Buenos Aires). Preliminary findings, seek to understand the obstacles encountered and find some signs of change in the way of operating urban politics.

\section{Keywords}

Municipal Policies, Territorial Policies. 
Formulación de políticas de ordenamiento territorial en municipios de México y Argentina a comienzos del siglo XXI: dificultades para su construcción

\section{PRESENTACIÓN}

Como tesis del ensayo se plantea que tanto en México, como en Argentina, en el proceso de construcción de las políticas de ordenamiento territorial municipal, se verifican características estructurales que conducen a dificultar su concreción, por lo que estas resultan no valoradas como políticas transversales al conjunto de acciones que tienen por objetivo el desarrollo de los ámbitos locales ${ }^{1}$.

En ambos países los municipios poseen responsabilidades en materia de ordenamiento territorial $^{2}$ y respecto de su articulación con el resto de políticas locales y de los otros niveles del Estado. Sin embargo, existen muchas dificultades para enfrentar estos compromisos, sus capacidades para formularlas e implementarlas son limitadas, más aun, de pensarlas como un instrumento fundamental para el desarrollo.

En municipios pequeños y medianos la situación es todavía más comprometida, ya que si bien las problemáticas podrían resultar de menor gravedad, y el desarrollo pareciese un objetivo más palpable, esta valoración del ordenamiento territorial aún resulta ajena a las prácticas cotidianas de la política. No puede decirse que la escala poblacional sea factor determinante de estas limitaciones, pero, al implicar diseños institucionales mínimos, escasa capacidad financiera y de gasto, e insuficiente disponibilidad de recursos puede afirmarse que en términos generales condiciona las capacidades al restringir fuertemente las acciones de los municipios (Ríos, RocCA y Bono, 2009).

En este marco, el objetivo del ensayo es indagar en el proceso de formulación de las políticas de Ordenamiento Territorial municipales, buscando identificar problemas, limitaciones y obstáculos que dificultan su concreción. Se ahondará en el conocimiento de esta problemática en dos municipios pequeños de ambos países: Tequisquiapan, ubicado en el estado de Querétaro, México, y Chascomús, situado en la provincia de Buenos Aires, Argentina (figuras 1 y 2). Ambos son municipios no metropolitanos y con avances similares en sus procesos de gestión territorial. En el cuadro 1 se sintetizan los principales indicadores sociales y económicos de ambos a manera de breve caracterización general.
1- El artículo es producto de la investigación desarrollada en el marco de la tesis para obtener el grado académico de Magister en Ciencias de Magister en Ciencias Dicho trabajo en elaboración Dicho trabajo en elaboración
se denomina "Politicas Locales de Ordenamiento Territorial en Latinoamérica: Evaluación y Perspectivas de Cambio en Municipios Pequeños y Medianos de México y Argentina a Inicios de Mexico y Argentina a Inicios
del Siglo XXI", dirigida por los arquitectos Néstor Omar Bono y María Julia Rocca.

2-En México a partir de la reforma de 1983 del Art. 115 constitucional se faculta a los municipios en materia de planeación del desarrollo urbano municipal. Otras normas que reglan el ordenamiento teritorial son Ley General de Asentam: la Ley General de Asentamientos
Humanos, la Constitución Política de los Estados y las leyes orgánicas municipales. En Argentina no existe ley a nivel nacional; si a nivel provincial, la Ley $8912 / 1977$. 
Cuadro 1: principales indicadores socioeconómicos de Tequisquiapan y Chascomús

\begin{tabular}{llll}
\hline Características & & Tequisquiapan & Chascomús \\
\hline Geográficas & Superficie & 343,6 & $4.225 \mathrm{~km} 2$ \\
& & & \\
\hline Población & Total & 54929 (2005) & 38.647 (2001) \\
& Ciudad Cabecera & 26858 (Tequisquiapan) & $\begin{array}{l}30670 \text { (Chascomús) } \\
9,1 \mathrm{hab} / \mathrm{km} 2\end{array}$ \\
& Densidad & & \\
& & & $4,48 \%$ \\
Ocupación & Sector Primario & $9,15 \%$ (año 2005) & $26,52 \%$ \\
PEA & Secundario & $49,10 \%$ & $66,51 \%$ \\
& Terciario & 39,62 & $2,44 \%$ \\
& Desconocido & $2,14 \%$ & $7,91 \%$ \\
\hline Económicas & PIB sector primario & Sin datos \% (2008) & $36,74 \%$ \\
& PIB sector secundario & $18,3 \%$ & $11,49 \%$ \\
& PIB Terciario (Comercio) & $45,3 \%$ & 43,85 \\
\hline
\end{tabular}

Fuente: elaboración propia según datos de INEGI e INDEC

Buscando responder al interrogante ¿cuáles factores facilitan o dificultan la etapa de formulación de las políticas de ordenamiento territorial?, se revisarán, en primer lugar, los aspectos conceptuales fundamentales para el entendimiento del nuevo rol del ordenamiento territorial en el contexto municipal. Luego se indagarán los principales instrumentos formulados en el marco de la política de ordenamiento en Tequisquiapan y Chascomús, haciendo hincapié en la viabilidad de su constitución como una política articuladora para el desarrollo. Finalmente se presentan algunas conclusiones preliminares, buscando sintetizar la valoración posible del ordenamiento territorial a partir de entender los obstáculos y encontrar algunos indicios de cambio en la forma de hacer las políticas.

\section{POLÍTICAS DE ORDENAMIENTO TERRITORIAL Y DESARROLLO DE LOS ES- PACIOS LOCALES}

Históricamente los municipios en México y Argentina han tenido un rol mínimo en cuanto a la formulación de políticas, en especial en relación con aquellas vinculadas con el desarrollo y el ordenamiento, concentrándose mayormente en funciones vinculadas con la 
obra pública y con la dotación de servicios, y han ido incluyendo en los últimos tiempos funciones vinculadas con la cuestión social, dejando en manos de los niveles superiores del Estado la responsabilidad en cuanto a políticas estructurales.

Este centralismo ha sido puesto en discusión durante las últimas décadas del siglo $\mathrm{XX}$, tanto en los ámbitos académicos como políticos, configurando un nuevo paradigma sobre el rol del municipio como ámbito de formulación de políticas. El contexto en el que comienzan a implementarse políticas con esta concepción estuvo dominado por el Consenso

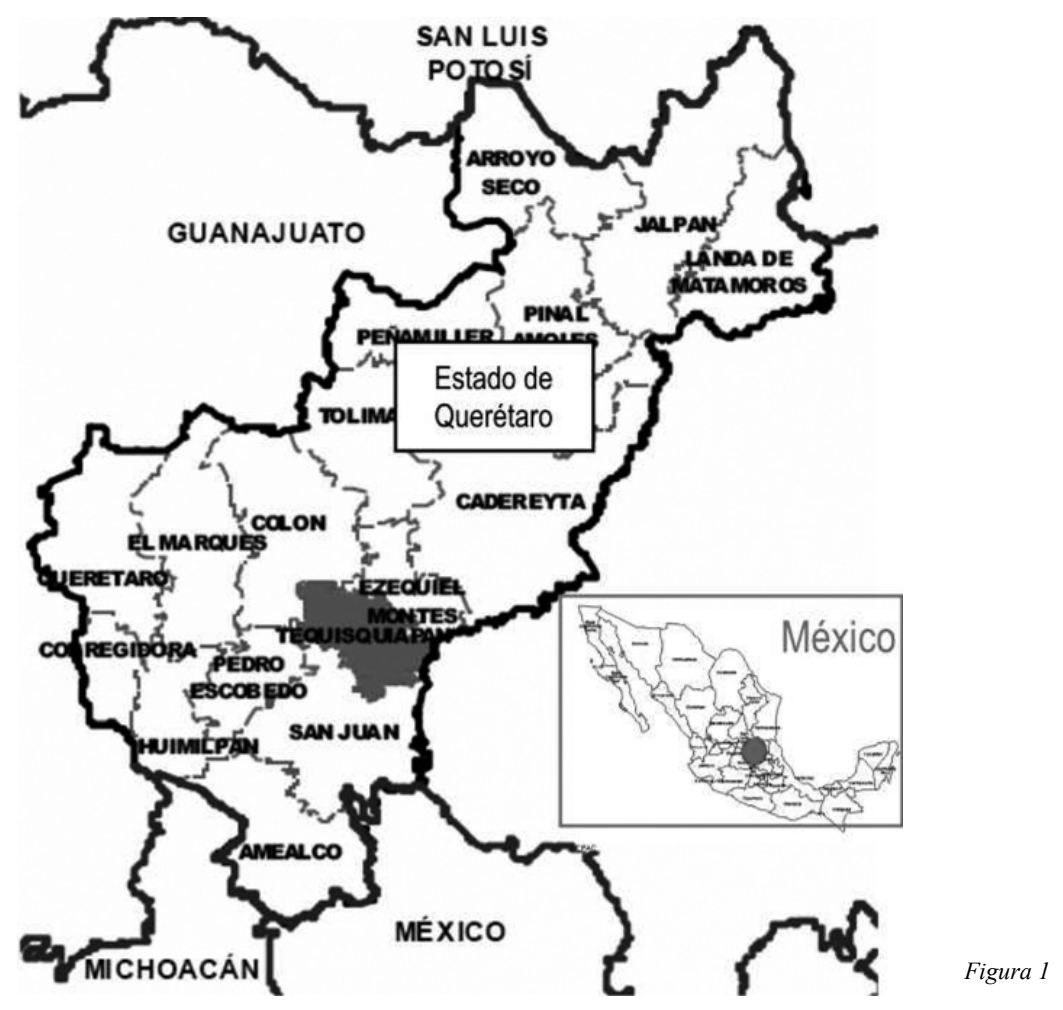




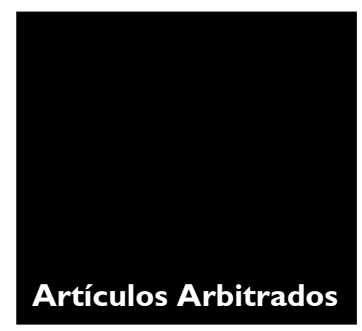

Licia Ríos

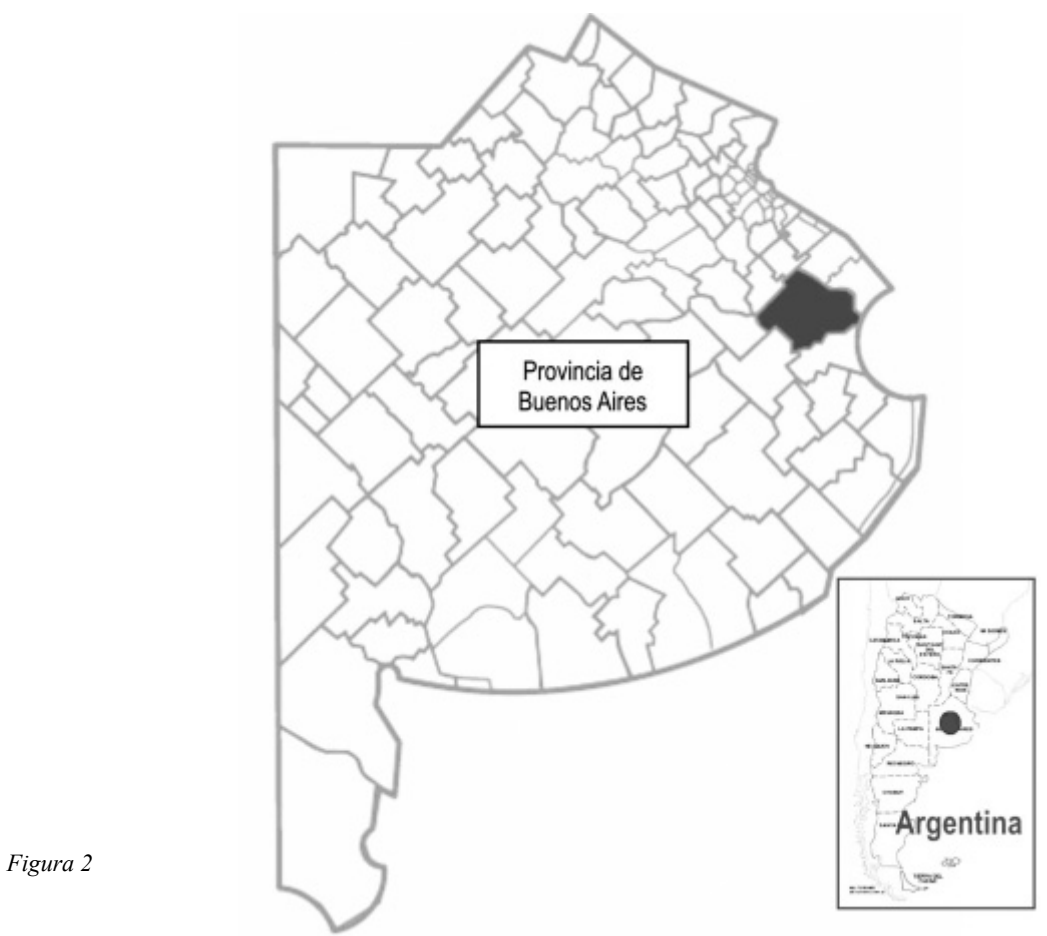

de Washington. Bajo este paraguas, desde los enfoques eficientistas de principios de la década del 90, englobados en lo que se llamó la Nueva Gestión Pública (OsBorne y GAEBLER, 1992), con la lógica de la reducción del Estado, se han transferido funciones hacia los gobiernos locales, con mínimos recursos. Las consecuencias sociales, económicas y territoriales del modelo neoliberal son bien conocidas y documentadas en infinidad de investigaciones.

Dentro de esta crítica al modelo del pensamiento único y a sus consecuencias, se inscribe un nuevo debate sobre cómo construir políticas desde los espacios locales, que coadyuven a la construcción de un nuevo modelo de territorio (local, regional, estatal y nacional). Estos nuevos enfoques revalidan al Estado como actor central en el proceso de desarrollo 

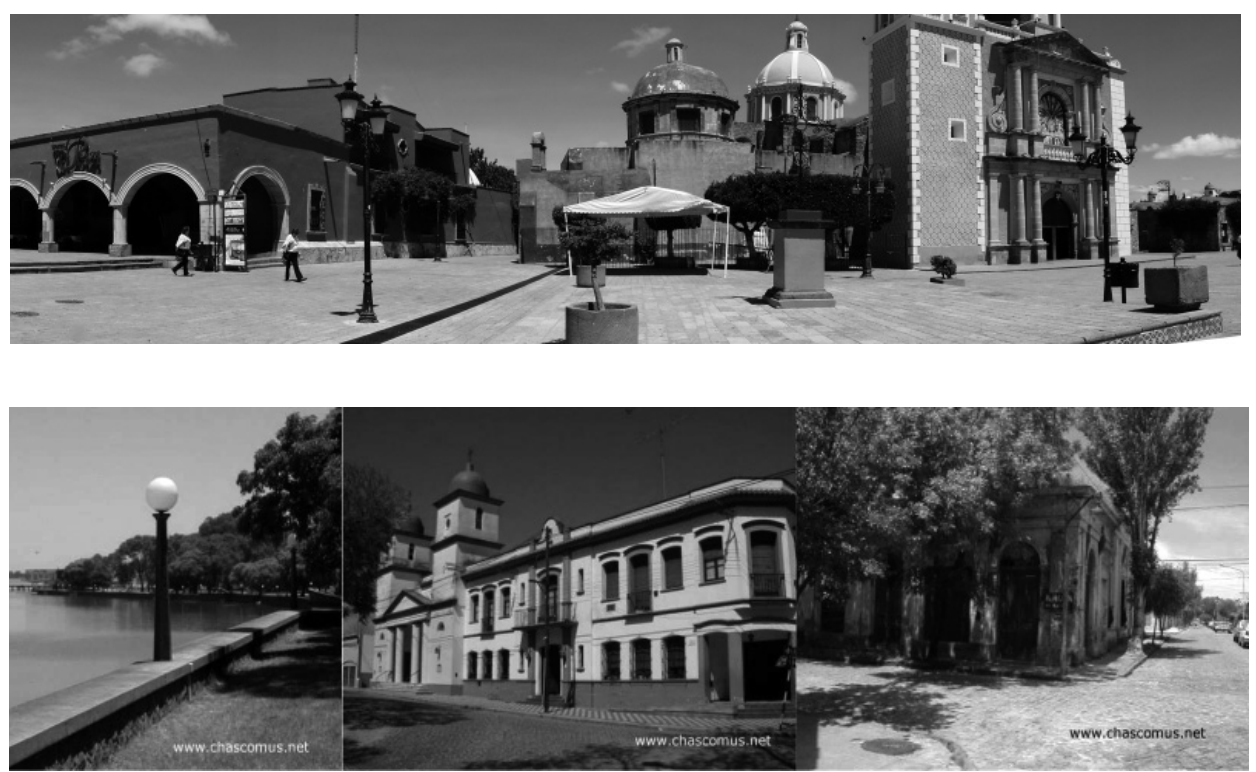

(EvANS, 2003) y proponen una "apertura participativa" de las administraciones públicas hacia una sociedad que ya no es vista como integrada por "clientes", sino por ciudadanos, y que cuenta tanto con plenos derechos, como con responsabilidades cívicas (SCIARA, et ál., 2007).

En este sentido, se refiere al desarrollo local como un proceso generado a partir de las capacidades endógenas, entendido como el conjunto de políticas impulsadas por los actores locales de un territorio que, sobre la base de un proyecto colectivo, buscan aprovechar bajo condiciones de sustentabilidad las potencialidades de un territorio, impactando positivamente en la economía y la sociedad, y cuya finalidad es satisfacer las necesidades humanas fundamentales y mejorar la calidad de vida de la población (MADOERY, 2008). Estas ideas resaltan la importancia de la cercanía, la interacción y la asociatividad como elementos cruciales que, relacionados con el territorio, favorecen la toma de decisiones y el "desarrollo local" (BoIsIER, 2003). 
3- Se utiliza el concepto de política pública como un programa de acción que una autoridad formal, en el marco de sus competencias, decide hacer o no (implica una positiva o simbólica, o inacción) en la búsqueda de coordinar los medios y disposiciones del Estado y las actividades privadas, para la realización de objetivos que son socialmente relevantes $y$ politicamente determinados.
En este marco, el ordenamiento territorial municipal adquiere nuevos roles, pasando de una visión espacialista a una integradora, ya que debe potenciar los recursos y capacidades locales no solo en el sentido tradicional (la ubicación geográfica, los recursos naturales, el ambiente, las infraestructuras, las empresas, los recursos científicos), sino que debe contribuir a la convergencia de objetivos, a veces divergentes, de la totalidad de las políticas sectoriales, para poder contribuir al desarrollo a largo plazo.

Para esta política, el territorio pierde el carácter pasivo propio de la visión sectorial para convertirse en estructurante de los objetivos, las políticas y las acciones públicas y privadas. Su fin es articular los objetivos económicos, sociales, ambientales y administrativos con el territorio, racionalizar las actuaciones sobre este y orientar previsoramente su desarrollo y aprovechamiento sostenible, basado, especialmente, en estrategias de uso, ocupación y manejo del territorio y de desarrollo territorial (OrTIZ y MASSIRIS, 1993).

Debe entenderse entonces el ordenamiento territorial como una política pública ${ }^{3}$ que expresa la voluntad de coordinar y armonizar las actuaciones con incidencia territorial de los diferentes niveles y sectores de la administración, que tiene como objetivo último garantizar el desarrollo sustentable para la calidad de vida de la población (CHABALGOITY, 2004). En el mismo sentido, las Políticas Territoriales, de Ordenamiento y Desarrollo son respuestas de la sociedad a los problemas y oportunidades del territorio, concebido a la vez como ámbito de la vida social y política, espacio de desarrollo y medio ambiente (DinOT, 1996) (figura 3).

Este reconocimiento del Ordenamiento Territorial como estrategia para el logro del desarrollo implica contar con una sólida organización institucional, jurídica e instrumental del conjunto de niveles de gobierno que posibilite su gestión y garantice la orientación y coordinación horizontal y vertical de las entidades participantes en el proceso, además de la integración de las políticas y planes sectoriales y territoriales. Esto implica que se requiere de tres cuestiones básicas para construir la arquitectura coherente para su desarrollo: una cobertura legal adecuada, otorgada por el marco jurídico; un desarrollo institucional para gestionar la formulación, implementación, control y seguimiento de la política y la formulación de instrumentos para conducir esta última, constituyendo los planes el instrumento básico de la ordenación del territorio.

De este conjunto de elementos y de la calidad de sus vínculos en el marco del sistema federal dependen en gran parte el carácter y los resultados de las políticas. El enfoque 
Formulación de políticas de ordenamiento territorial en municipios de México y Argentina a comienzos del siglo XXI: dificultades para su construcción

teórico, naturaleza e instrumentos de las políticas necesariamente se ven delimitados por tal combinación de factores: es así que en el marco de un esquema de gobierno con carácter fuertemente centralista pareciera más difícil alcanzar políticas de ordenamiento locales con fuerte sustento territorial, mientras que un sistema que privilegie el federalismo necesariamente impregna de mayor relevancia a los municipios y a "lo local", como actores del desarrollo de sus territorios. De no existir este marco articulado y esta valoración y especificidad de cada nivel del Estado, es posible que se exponga a los municipios a la sola elaboración de planes de ordenamiento ajenos al contexto general.

Aunque la responsabilidad del ordenamiento territorial corresponde a la administración pública, será por lo antedicho que la participación social se constituye en un instrumento fundamental para el Ordenamiento Territorial municipal. Una verdadera apertura a la participación pública auspicia una mayor legitimidad a la política, que seguramente redundará en una mejor y adecuada implementación y evaluación.

Esto implica que la participación tenga a la vez carácter informativo como de insumo para la concertación de objetivos, la construcción de diagnósticos más cercanos a la realidad local, y de estrategias afines para su consecución. Es decir, no debe quedar regulada solo por la definición y aprobación del modelo de ciudad, sino que debe estar ligada con un sistema continuo de información pública sobre las transformaciones que se producen en el territorio municipal y sus verdaderas consecuencias, que permita, en paralelo, generar mecanismos de acción pública ágiles y eficientes para demandar a las administraciones públicas el cumplimiento de contenidos, obligaciones y compromisos (SERRANO, 2004). Es decir, la participación no debe terminar con el proceso de elaboración de los instrumentos.

Sin embargo, avanzada ya la primera década del siglo XXI, la mayoría de los municipios mexicanos y argentinos evidencian dificultades para avanzar hacia instancias de mayor preponderancia en cuanto a la formulación e implementación de políticas de desarrollo y ordenamiento territorial. Para encontrar algunas causas de estas dificultades se propone observar a continuación las primeras fases del proceso de las políticas de ordenamiento en los dos municipios seleccionados de México y Argentina, entendiendo que estas acciones públicas pueden analizarse reconociendo componentes lógicamente necesarios e interdependientes integrados a manera de proceso $^{4}$ (Aguilar Villanueva, 2000).
4- AGUILAR VILLANUEVA (op. cit.) plantea para el estudio de las políticas públicas un proceso integrado por varias y complejas etapas (Análisis de los Problemas Públicos (Analisis de los Problemas Publicos y Agenda de Gobierno la "La Hechura de las Políticas"
(o formulación) y "La Implementación de las Políticas"). De ninguna manera se trata de eventos sucesivos y separables. 
$\overline{5-E n ~ Q u e r e ́ t a r o ~ t o d o s ~ l o s ~}$ municipios deben contar con un Plan Municipal de Desarrollo según lo establecido en la Ley de Planeación del Estado de Querétaro (2008). Alli se establece la finalidad de la planificación del desarrollo, el Sistema Estatal de Planeación para el Desarrollo, los órganos de planeación, el sistema de concertación social y responsabilidades.

6- El código Urbano del Estado de Querétaro (1992) establece que la ordenación y regulación de los asentamientos humanos se llevará a cabo en los ámbitos locales a partir de los Planes Municipales de Desarrollo Urbano.

\section{LIMITACIONES Y OBSTÁCULOS EN LA ETAPA DE FORMULACIÓN DEL ORDE- NAMIENTO TERRITORIAL EN TEQUISQUIAPAN Y CHASCOMÚS}

En este apartado se indagará la etapa de formulación de las políticas recientes y vigentes municipales de ordenamiento territorial, buscando en primer lugar reconocer sus principales aspectos teóricos y metodológicos, en función de profundizar la existencia (o no) de una valoración integral del territorio; para luego analizar los aspectos técnicos fundamentales que coadyuven a tal valoración.

$\mathrm{Al}$ analizar estos aspectos, se ahondará en su naturaleza, buscando sintetizar los enfoques que las guían; la posibilidad de articulación entre actuaciones sectoriales, observando la consideración de una organización institucional para propiciarla; su legitimación social, considerando la participación en la reivindicación pública de la política; y finalmente, la coherencia del esquema metodológico con estas pautas. Finalmente se profundizará en el conocimiento de la previsión de su instrumentación para lograr los objetivos y escenarios propuestos.

Antes de avanzar en el análisis, se presentarán brevemente los instrumentos considerados para proceder a evaluar los principales formulados por las políticas.

\section{Los instrumentos analizados}

Como se ha dicho, el estudio se realizará sintetizando un conjunto de instrumentos que conforman "la política de ordenamiento territorial" de ambos municipios, ya que en ambos casos no existe una política explicitada como tal en un instrumento que le otorgue formalidad y compromiso, sino que se trata un conjunto de instrumentos (incluso formulados en períodos muy distantes entre sí) que conforman el programa de acción municipal de ordenamiento territorial (cuadro 2).

En Tequisquiapan los instrumentos registrados como componentes de la política territorial son el Plan Municipal de Desarrollo 2006-2009, principal instrumento de la planificación municipal que establece los propósitos, objetivos, prioridades, estrategias generales y criterios de acción para el desarrollo integral del municipio ${ }^{5}$; el Programa Municipal de Desarrollo Urbano de Tequisquiapan ${ }^{6}$, cuyo fin es atender el ordenamiento territorial de la ciudad cabecera en particular y del sistema de asentamientos humanos del resto del municipio en general, y el Plan Sectorial de Desarrollo Urbano Turístico, instrumento para la regulación del sector turístico, principal actividad del municipio. 
Formulación de políticas de ordenamiento territorial en municipios de México y Argentina a comienzos del siglo XXl: dificultades para su construcción

\section{Cuadro 2: Instrumentos de Planificación que conforman la Política de Ordenamiento Territorial de Tequisquiapan y Chascomús}

\begin{tabular}{|c|c|c|c|}
\hline \multicolumn{2}{|c|}{ Tequisquiapan } & \multicolumn{2}{|c|}{ Chascomús } \\
\hline Instrumento & Тіро & Instrumento & Тіро \\
\hline $\begin{array}{l}\text { Plan Municipal } \\
\text { de Desarrollo 2006-2009 }\end{array}$ & Plan de desarrollo & $\begin{array}{l}\text { Plan de Desarrollo, } \\
\text { Territorial (PDT) } \\
\text { (en estudio) }\end{array}$ & Proyecto normativo \\
\hline $\begin{array}{l}\text { Plan Sectorial de Desarrollo } \\
\text { Urbano Turístico de } \\
\text { Tequisquiapan (2007) }\end{array}$ & $\begin{array}{l}\text { Normativo para el } \\
\text { Desarrollo de la } \\
\text { actividad turística }\end{array}$ & $\begin{array}{l}\text { Lineamientos Estratégicos } \\
\text { de Desarrollo Local-: } \\
\text { Documento Básico } \\
\text { de Diagnóstico } \\
\text { (LEDL) (2005) }\end{array}$ & $\begin{array}{l}\text { Documento base } \\
\text { de diagnóstico } \\
\text { para el desarrollo }\end{array}$ \\
\hline $\begin{array}{l}\text { Plan de Desarrollo Urbano } \\
\text { del Centro de Población } \\
\text { de Tequisquiapan } \\
\text { (PDUCP), (2005) }\end{array}$ & $\begin{array}{l}\text { Normativo de usos } \\
\text { del suelo }\end{array}$ & $\begin{array}{l}\text { Código de Zonificación } \\
\text { (CZP), (1981 } \\
\text { y modificatorias) }\end{array}$ & $\begin{array}{l}\text { Normativo de usos } \\
\text { del suelo }\end{array}$ \\
\hline
\end{tabular}

Fuente: elaboración propia

Por su parte, los instrumentos analizados en el municipio de Chascomús son tres, uno de carácter normativo, mientras que los otros dos aún carecen de vigencia legal. El primero, el Código de Zonificación ${ }^{7}$ según usos del suelo (Ordenanza vigente $N^{0}$ 1272/1981), en el marco del proceso de planificación preceptuado en el Decreto Ley 98912/1977, tiende a cubrir las necesidades mínimas de ordenamiento físico territorial, determinando la estructura general del espacio municipal, la de cada una de sus áreas y zonas componentes, estableciendo normas de uso, ocupación y subdivisión del suelo, dotación de infraestructura básica y parámetros morfológicos. Los otros instrumentos, el Plan de Desarrollo Territorial y su documento de Diagnóstico, surgidos mediante un proceso de planificación estratégica local, vienen a sustituir y completar el anterior, aún no fueron promulgados, aunque algunas de las acciones por este proceso ya han sido implementadas.

No es el propósito analizar íntegramente estos documentos, sino considerar en profundidad los aspectos clave que en su fase de formulación pueden implicar obstáculos para lograr una adecuada implementación y evaluación.

\footnotetext{
una adecuada implementación y evaluación.
}

7- El Decreto Ley 8912/1977 establece las distintas etapas del proceso de ordenamiento territorial. Ellas son la Delimitación Preliminar de Áreas, la Zonificación Urbana y el Plan de Ordenamiento Territorial. Chascomús se encuentra recién cursando la segunda instancia prevista por esta ley, la Zonificación Urbana. 


\section{Naturaleza}

Desde la perspectiva planteada, el ordenamiento territorial tiene carácter multidimensional en tanto debe conciliar aspectos económicos, sociales y físico ambientales planteando las estrategias de uso y ocupación del territorio de acuerdo con sus potencialidades y problemáticas. Por su naturaleza deberá involucrar e interrelacionar efectivamente a todas las dimensiones del territorio, a fin de obtener resultados que permitan su gestión adecuada y el desarrollo sostenible a largo plazo.

Del análisis del caso de Tequisquiapan debe rescatarse en primer lugar que los tres instrumentos analizados buscan integrar las políticas en el desarrollo territorial sustentable, aunque en particular adquieren diferentes visiones, y en segundo lugar que tienen plena validez legal, tanto por su aprobación a nivel local como estatal.

El Plan de Desarrollo Urbano es un instrumento enfocado hacia desarrollo territorial que considera la transversalidad e intersectorialidad del ordenamiento territorial para alcanzar los objetivos del desarrollo sustentable en el largo plazo, tanto porque se explicita tal vocación, como porque toma en consideración aspectos socioeconómicos, culturales y físico ambientales, políticos y administrativos, tanto en su nivel diagnóstico como estratégico, programático e instrumental. También tiene un carácter multidimensional e integral el Plan de Desarrollo Turístico.

Paradójicamente no ocurre lo mismo con el Plan Municipal de Desarrollo, cuya planificación está más cerca de la visión sectorial, ya que se trata de un conjunto de acciones planificadas por las distintas áreas de gobierno, no explicitándose sus vinculaciones, como tampoco con las políticas vigentes de períodos anteriores, por lo que difícilmente pueda adquirir el sentido estratégico que debiera tener cualquier instrumento de este tipo.

En relación con la escala de los instrumentos se corrobora que hay una consideración de las escalas urbana-rural-regional, tanto en la fase diagnóstica como de propuesta de lineamientos y proyectos en el caso del Plan de Desarrollo Urbano y en el Plan de Desarrollo Turístico. No puede afirmarse lo mismo en el caso del Plan de Desarrollo, por el énfasis sectorial que adquiere y el escaso desarrollo que alcanzan tanto los diagnósticos como las proposiciones.

Por las características de los tres instrumentos citados, puede inferirse preliminarmente que es factible la constitución en Tequisquiapan de la política de ordenamiento como integradora del resto de políticas territoriales, en tanto adquiere un rango superior integrador por su cualidad multidimensional. 
En el caso de Chascomús, es necesario resaltar en primer lugar que coexisten instrumentos sustentados por dos paradigmas distintos de planificación, el tradicional, con el que se identifica al Código de Zonificación, y la planificación estratégica, que da marco a los otros dos instrumentos, el Diagnóstico y el Plan de Desarrollo Territorial, resultantes de un proceso participativo para el desarrollo local, construidos en fecha más reciente.

En consecuencia, ambos grupos de instrumentos no tienen conexión y articulación, sus modalidades y planteamientos generales no coinciden. El Código de Zonificación es un instrumento unidimensional, que toma en cuenta principalmente cuestiones espaciales, parte de una situación problemática de origen (1980) que no asume cambios y nuevas tendencias en su proceso, y la planificación se da en un momento inicial desde el que se plantea un esquema rígido. Por su parte, los instrumentos resultantes del proceso estratégico obedecen a objetivos vinculados con el logro del desarrollo sustentable para el mejoramiento de la calidad de vida de la población, y por el propio carácter metodológico, su perfil es dinámico, procesal y participativo.

Sin embargo, en este último se aprecian diferencias sustanciales en cuanto a la diversidad de elementos involucrados, ya que el proceso estratégico en su fase diagnóstica está construida desde una perspectiva integral y participativa que incluye aspectos de medio natural, económico, patrimonial, sociocultural, mientras que la fase propositiva sólo tiene carácter integral en la definición de sus objetivos y estrategias de intervención, sobre los que se sustenta un conjunto de proyectos de intervención física y la zonificación reglamentaria.

Por su parte, en cuanto a la legalidad de los instrumentos, si bien la planificación estratégica goza de un importante prestigio masivo, los instrumentos resultantes no consiguieron hasta el momento ser reglados y pactados para el interés general otorgándoles seguridad jurídica. Sólo tiene vigencia legal el Código de Zonificación.

En cuanto a la escala de los instrumentos formulados, en el caso de la zonificación se trata de uno municipalista a ultranza, aunque limitado sólo al espacio urbano, mientras que el plan de desarrollo territorial incluye un diagnóstico y propuestas a escala regional y del partido y un proyecto normativo para el ámbito de su jurisdicción.

Esta desarticulación entre instrumentos y la diferencial apreciación del territorio, sus recursos y actores permite interpretar preliminarmente que la política de ordenamiento en Chascomús está guiada por una interpretación espacialista del territorio y funcional de la 
sociedad, en tanto la validez legal ha sido alcanzada solo por el Código de Zonificación. No obstante, puede advertirse la apertura hacia procesos más integrales, en tanto la legitimidad alcanzada por el proceso de planificación estratégico adquiera un instrumento normativo acorde, que lo jerarquice como política y le otorgue validez legal y perdurabilidad.

Puede sintetizarse que, en términos generales, en ambos casos existe voluntad de concebir el ordenamiento territorial como una política transversal al resto de las políticas públicas. A pesar de las diferencias explicitadas, puede leerse que hay cierta confluencia de propósitos orientados hacia el desarrollo territorial, aunque mayormente expresada en forma de objetivos. Esto en concordancia con una revalorización explícita del rol municipal en la conducción del desarrollo y el ordenamiento. No obstante, cuando se avanza hacia instancias propositivas, en ambos municipios el reconocimiento del territorio dualiza entre lo urbano y lo rural, lo municipal de lo regional, lo físico de las actividades económicas, etc.

\section{Coordinación}

La complejidad de la política de ordenamiento demanda coordinación para lograr conciliar aspectos físicos, económicos y sociales, y para que adquiera su cualidad multidimensional y participativa, evitando contradicciones y reduciendo disfunciones que impidan o dificulten su proceso. Para ello será fundamental la capacidad de propiciar la articulación entre las actuaciones de las distintas dependencias y niveles del Estado, y en donde cada uno realice tareas en relación de cooperación horizontal con los otros organismos participantes, como también entre las instituciones políticas y la sociedad civil. En este apartado se ahondará en la identificación de contenidos, procesos y diseños capaces de favorecer la coordinación para colaborar en que dicha complejidad y posibles divisiones alcancen unidad y simplificación.

De la misma manera que en la variable anterior, en Tequisquiapan se observa una mayor atención a la coordinación en el Plan de Desarrollo Urbano, mientras que en el resto de los instrumentos no se hace alusión a la existencia de procesos o diseños capaces de propiciar la articulación, factor que deja entrever una posible sectorialidad asociada con áreas específicas de gobierno.

Como se ha visto, en el Plan de Desarrollo Urbano, tanto el diagnóstico como las propuestas interrelacionan las distintas dimensiones del ordenamiento territorial, medio físico, medio ambiente, medio económico y social, y su articulación en las distintas escalas. Para ello, se toman en consideración las vinculaciones en términos de contenidos dentro 
del Estado Municipal y el marco de los objetivos y acciones de los niveles de gobierno superior e intermedio, estableciendo también los distintos grados de corresponsabilidad sectorial y de origen de recursos para materializar las acciones.

Sin embargo, esta consideración no implica necesariamente que la política de ordenamiento se materialice en un contexto donde prevalezcan las articulaciones de tipo horizontal, donde todos contribuyen de igual forma a la consecución de los objetivos del desarrollo en general y del ordenamiento territorial en particular, ya que si se vuelve a observar la distribución interna entre direcciones, es posible que al interior del municipio se establezcan relaciones jerárquicas que atentan contra la coordinación en general. También son posibles disfunciones en las relaciones con los órdenes de gobierno superiores, primando la coordinación de tipo jerárquica.

En el caso de Chascomús, como consecuencia de la disímil naturaleza de cada uno de los instrumentos que conforman la política, la coordinación adquiere desigual alcance, siendo nula su previsión en el Código de Zonificación, y solo a través de la participación social en los instrumentos surgidos del proceso estratégico, sin prescripción de modalidades de articulación desde el punto de vista organizacional.

Si bien la fase diagnóstica del Plan de Desarrollo Territorial está construida desde una perspectiva integral y participativa, incluso de la que formaron parte distintos sectores de la administración local, intermedia y superior, relacionadas con el financiamiento y asistencia a proyectos, no ocurre lo mismo con la fase de formulación de estrategias de intervención, en la que no se especifican organismos, mecanismos o instrumentos que contribuyan a que las acciones de las distintas partes se enlacen coherentemente para la obtención de los objetivos establecidos. Tampoco se menciona la articulación con otras instancias preexistentes de asociativismo intermunicipal, más aun considerando que existen entes regionales en los cuales el municipio se encuentra involucrado, como el Consorcio de Municipios Turísticos (COTAB) y el Consorcio Productivo del Salado (COPROSAL). Esto permite inferir que estas relaciones entre municipios pueden adolecer de limitados alcances, no logrando en este caso integrarse con el principal instrumento de planificación del ordenamiento territorial municipal, para alcanzar la cooperación y coordinación a nivel de las acciones concretas que logren ampliar "lo local" más allá de "lo municipal".

En resumen, en los dos casos analizados se observa falta de previsión de mecanismos e instrumentos para la coordinación de la política, lo que puede conducir a consolidar una forma de gobernar sectorial y centralizada, a contradicciones de objetivos y 
acciones entre actuaciones con incidencia territorial y superposición e incoherencia; cuestiones que se vuelven más problemáticas si se considera que el área encargada del ordenamiento territorial cuenta con un limitado desarrollo dentro de las estructuras organizativas municipales.

\section{Legitimación social}

Se ha visto que el ordenamiento territorial puede legitimarse si es capaz de insertarse entre los procesos y actores que modelan la realidad local y articularlos racionalmente para alcanzar los objetivos de transformación. Esta participación social permite considerar opiniones e intereses de los diferentes actores para lograr acuerdos, propósitos, objetivos y metas compartidas en cuanto al desarrollo y al ordenamiento del territorio. La construcción en este sentido de la legitimidad pública de la política ciertamente redundará en mayores posibilidades de una adecuada implementación y evaluación.

Con este sentido se revisará a continuación el grado de discusión y concertación logrado en el proceso de formulación de la política de ordenamiento municipal en los municipios seleccionados, a partir de reconocer procesos, instrumentos o mecanismos de participación.

En Tequisquiapan, a pesar de que se propicia la concertación entre actores para construir diagnósticos, elegir las prioridades y favorecer el seguimiento de las obligaciones adquiridas por el municipio, solo se explicita su utilización abierta en la construcción de las fases diagnósticas de cada uno de los planes, adquiriendo forma selectiva en la implementación.

La mayor explicitación de la participación se refiere en los casos del Instituto Municipal de Desarrollo Urbano, conformado por especialistas de organismos del sector público, privado y social, cuyo fin es la asistencia en amplios temas técnicos vinculados con el desarrollo urbano; y de la Agencia de Infraestructura Turística, constituida por actores vinculados con la actividad, cuyo objeto es la coordinación, evaluación y seguimiento del plan.

Sin embargo, otorgar protagonismo solo a algunos agentes estatales y privados implicados, puede conducir a una observación sesgada de la realidad y restar operatividad al plan, e incluso limitar la duración a sólo al período de gobierno que la formuló, ya que la población no se ha apropiado de ella. Y particularmente la gran cantidad de competencias otorgadas al Instituto Municipal de Desarrollo Urbano puede conducir a que se minimice el rol de la dirección encargada del ordenamiento territorial (ya minimizado en cuanto 
a jerarquía y recursos otorgados según lo visto en el análisis de la fase de formulación), o a una superposición de competencias entre ambas, y también limitar su estabilidad en caso de disolución de esta institución creada al margen del organigrama municipal.

En Chascomús, la participación social no está considerada el principal instrumento normativo vigente, y en el caso de los formulados mediante el proceso de planificación estratégica, paradójicamente se ha limitado a la fase de diagnóstico, que sirve de puntapié inicial para el Plan de Desarrollo Territorial.

En el Código de Zonificación, la participación en forma articulada no ha sido sostén de su formulación, contrariamente a la voluntad expresa del Decreto Ley 8912/1977 de que esta se instituya en instrumento básico del ordenamiento territorial municipal. Durante sus 28 años de vida, esta norma ha sido modificada gran cantidad de veces, a pedido de casos individuales que requirieron una actualización respondiendo en general a la necesidad de expansión urbana de la ciudad, por lo que la participación de la sociedad se ha dado en forma individual, en atención a objetivos también particulares.

En el diagnóstico construido en el proceso de planificación estratégica la participación se revela ante la necesidad de generar una base de sustentación para consensuar y legitimar las políticas para el desarrollo local, habiendo sido el origen de la iniciativa el gobierno municipal, aunque sustentado metodológicamente por grupos asesores externos. Según lo expresado en el documento diagnóstico, el grado de participación ha sido amplio y abierto, con la presencia de organizaciones sociales, políticas, empresarias y religiosas, involucradas mediante procesos de comunicación y formación. También constituye una herramienta de valor para favorecer la participación a través de la comunicación la construcción de un sistema de información municipal, que a su vez serviría para transparentar $\mathrm{y}$ dar eficiencia al proceso.

Por su parte, en el documento del Plan de Desarrollo Territorial no se explicitan los resultados de ningún proceso concertado que lo sustente, constituyéndose en un menoscabo al proceso participativo del que es resultado. Tampoco se hace mención a instancias de validación participativa de las propuestas concretas de esta instancia. Merece destacarse también que se prevé entre otros instrumentos para su implementación la creación de un Consejo de Ordenamiento Urbano y Territorial, como un organismo de participación selectiva de actores sociales considerados oportunos para la asistencia técnica en esta fase. 
Esta heterogénea apreciación de la participación social y desarticulación entre fases demuestra que en términos generales el proceso participativo ha tenido una complejidad que ha acabado siendo muy baja, en tanto ha servido solo para construir acuerdos en la fase diagnóstica, para comunicar sus resultados y construir sistemas de información para la toma de decisiones, pero no ha sido sostenido en el tiempo.

En síntesis, para ambos casos puede afirmarse que a pesar de la enunciación y concreción de ciertas acciones participativas, adquieren escasa importancia, ya sea por su limitado desarrollo en la formulación de los principales instrumentos de planificación, basada mayormente en una participación de carácter consultivo, como por la restringida estabilidad de los procesos participativos.

\section{Carácter planificado}

Se ha afirmado que es inherente a toda política de ordenamiento territorial su carácter planificado, en tanto le confiere coherencia al conjunto de acciones, constituyéndose su elaboración conceptual y metodológica en el Norte de todo el proceso. De este modo, toda política de ordenamiento debiera comenzar con una ajustada evaluación del territorio que permita conocer y explicar los procesos territoriales, a fin de valorar los problemas que hay que corregir y potencialidades que hay que desarrollar, como también las reales posibilidades de modificar positivamente la situación. En la planificación del proceso también será necesario precisar las herramientas para conseguir los resultados en las distintas etapas, y del mismo modo un sistema permanente de seguimiento y control, los responsables, tiempos y recursos, entre otros aspectos. Se observará aquí el encadenamiento de este conjunto y su coherencia.

En Tequisquiapan, en términos generales el conjunto de instrumentos manifiesta un esquema metodológico coherente tanto en su conjunto, como en su interior, siendo menos riguroso el caso del Plan Municipal de Desarrollo.

Tanto el Plan de Desarrollo Urbano Turístico, como el de Desarrollo Urbano presentan un esquema coherente que busca articular fielmente los diagnósticos con las fases propositivas, y considerar en todo momento la naturaleza de la visión integral del territorio. Ambos plantean un importante avance en cuanto a la definición de los instrumentos para implementar la política, ya sean programas o proyectos, la definición de sus costos y el involucramiento de distintas instituciones y actores sociales para la complementación de acciones.

También se da importancia a esta planificación en el largo plazo, con previsiones para el presente, el corto, el mediano y el largo plazo, e insertas en el contexto estatal y nacional, 
aunque no se plantean claramente en forma de escenarios, sino de concreciones a través de proyectos de obras.

En términos generales en el caso de Chascomús, el distinto sustento teórico de los instrumentos y los desfases temporales en su conjunto son manifestaciones de la incompatibilidad metodológica de los componentes de la política, lo cual no contribuye a que esta alcance un mínimo carácter planificado.

En este caso, el carácter dinámico que debiera manifestar el Diagnóstico para el Desarrollo Local no es finalmente el alcanzado. El desfase temporal entre la realización del diagnóstico y la propuesta, que aún se encuentra en discusión inmoviliza la realidad territorial al año 2005 , contrariamente con lo que debiera ser un proceso continuo y dinámico, capaz de incorporar los distintos procesos territoriales que van sucediendo.

También existe una desarticulación en la planificación del proceso entre ambas etapas en lo referente a sus contenidos; en tanto el diagnóstico no sostiene la propuesta en sus valoraciones y objetivos, este último documento desvaloriza mayormente la inicial visión integral adquiriendo un carácter más cercano al de una zonificación. Si bien se denomina Plan de Desarrollo Territorial, y se sustenta en amplios objetivos y estrategias, no existe un plan que los oriente, otorgue contenido sólido y firme y exprese la forma de su concreción; sólo se incluye una zonificación para alcanzarlos.

Este desajuste entre los dos instrumentos surgidos del proceso de planificación estratégica conduce a que finalmente se interpreten los fenómenos y procesos territoriales desde una única disciplina, y de carácter espacial, contrariamente a las búsquedas ideológicas y metodológicas del proceso de planificación del que surgen, que lleva implícitas una aproximación multidisciplinar, una aspiración a la coordinación e integración territorial de las políticas y la asunción de la planificación como un proceso dinámico, pero necesario.

En resumen, se puede afirmar que en ambos grupos de instrumentos existe una diferencial coherencia metodológica. Mientras que de la lectura conjunta de los instrumentos del caso mexicano se evidencia un avance hacia una concepción del ordenamiento en términos de integralidad y con carácter planificado, en Chascomús se evidencia una desarticulación entre los principales instrumentos de la política de ordenamiento, no sólo por su incoherencia metodológica, sino también por los desfasajes temporales entre ellos. 


\section{Previsiones para su instrumentación}

Toda política requiere de la definición de un camino para alcanzar los objetivos y escenarios propuestos; por ello en la implentación del ordenamiento territorial se requiere de la utilización eficiente de un conjunto de instrumentos mediante los cuales sea posible su logro en las distintas instancias del proceso. Considerando la importancia de esta atención, en este apartado se revisará la previsión de herramientas, observando su coherencia con los aspectos teóricos y metodológicos valorados en el apartado anterior y en su conjunto.

En el caso de Tequisquiapan, la instrumentación de la política de ordenamiento es desigual por instrumentos, siendo el Plan de Desarrollo Urbano Municipal el que mayores previsiones despliega.

El Plan Municipal de Desarrollo carece de instrumentos para efectivizarlos, mientras que en el Plan Sectorial de Desarrollo Urbano Turístico sólo se prevé como principal instrumento operativo la creación de una Agencia de Infraestructura. Por su parte, el Plan de Desarrollo Urbano Municipal incluye un extenso número de herramientas para realizar los propósitos, políticas y estrategias (de planificación, sistema de información demográfica, de regulación, de fomento y administrativos). Sin embargo, muchos son objetivos más que instrumentos propiamente dichos.

En el caso de Chascomús, la previsión de instrumentación de la política de ordenamiento es mínima, en tanto el Código de Zonificación es considerado en sí mismo como el principal y único instrumento para la planificación del territorio, mientras que las previsiones establecidas en la planificación estratégica no se adecuan a las aspiraciones integrales que requiere tal proceso, como tampoco a las definiciones sectoriales propuestas.

El Plan de Desarrollo Territorial es el único que enuncia mecanismos para viabilizar sus objetivos y estrategias. Si bien poseen un alto valor desde el punto de vista teórico, ya que abarcan distintos aspectos del proceso de implementación (planificación, promoción, gestión del suelo, participación, etc.), tanto en forma conjunta como individual carecen de especificidad al no estar vinculados con las estrategias u objetivos. A diferencia de la generalidad de esta identificación de instrumentos, se destaca la construcción de un Banco de Información Municipal, sistema de información utilizado en la fase diagnóstica del plan que hubiera podido haber resultado adecuado para medir la evolución de algunos indicadores en su proceso de evaluación. Sin embargo, en la propuesta no se hace mención de este sistema. 


\section{Del análisis de los casos Chascomús y Tequisquiapan debe señalarse que la escasa previsión de instrumentación de las políticas puede limitar en la voluntad de los ejecutivos locales la ejecución de la política.}

\section{CONCLUSIONES}

Se ha visto la emergencia de nuevas discusiones y búsquedas de acción política (incluso normativas) que ponen en discusión la concepción centralista encontrando en el municipio un nuevo ámbito de formulación de políticas, y donde el Estado es un actor central del proceso de desarrollo.

En este contexto el ordenamiento territorial es una política fundamental para lograr los objetivos del desarrollo, en consideración de su carácter integral y horizontal. En este sentido, se rescata la doble intención de preparar al territorio para facilitar su implementación y de velar para que estas no conduzcan a minimizar las potencialidades del territorio (Chabalgoity, 2004).

Sin embargo, a lo largo del ensayo, que permitió estudiar dos casos de municipios de México y Argentina, se ha podido comprobar la existencia de características estructurales en la formulación de las políticas de ordenamiento territorial municipales que podrían dificultar o limitar sus posibilidades de concreción. Estos obstáculos - teóricos, metodológicos y técnicos - llevan implícita de algún modo la falta de valoración del ordenamiento territorial como política transversal, o eje articulador, del conjunto de acciones que buscan el desarrollo de los ámbitos locales.

En primer lugar es importante destacar que en ninguno de los casos analizados se ha formulado una política de ordenamiento territorial, sino que se trata de un conjunto de instrumentos que conforman el programa de acción municipal, verificándose incluso la coexistencia de distintos enfoques, producto de las grandes brechas temporales ocurridas entre la formulación de cada uno.

Se han indagado distintos aspectos vinculados con el sustento teórico y metodológico de las políticas, observando la valoración del territorio. Allí se ha observado la emergencia de un proceso de transformación en el enfoque de las políticas que se traslada desde una visión tradicional y espacialista de la planificación física hacia una que tiene como objetivo 
el desarrollo territorial, con lo cual se ha verificado la coexistencia de planes de desarrollo y zonificaciones, formas de actuación casuística y estratégica, participación institucionalizada y falta total de concertación, entre otras contradicciones.

En lo referente a la existencia mecanismos que propicien la articulación de las distintas actuaciones sectoriales se ha visto la voluntad de concebir el ordenamiento territorial como una política transversal al resto de las políticas públicas municipales y también se ha reconocido la valoración de la integración en sentido vertical con los distintos niveles del Estado. Sin embargo, estas aspiraciones aún no se ven reflejadas en la totalidad de los instrumentos analizados, que reflejan la continuidad de la visión sectorial de las políticas, que prioriza la planificación de los territorios urbanos y se sujeta a los límites jurisdiccionales.

Por otra parte, si bien la participación social es un instrumento necesario tanto para sustentar la territorialidad de las políticas como para construir su legitimidad, y a pesar de su repetida valoración, es limitada la importancia que se le ha otorgado considerando el conjunto de instrumentos que conforman ambas políticas.

En cuanto a la coherencia del proceso metodológico, necesaria para conseguir avanzar de los objetivos hacia hechos concretos, resulta muy diferente lo sucedido en Tequisquiapan, donde hay rasgos de coherencia entre el objetivo del desarrollo sustentable deseado y el proceso metodológico utilizado, de lo visto en Chascomús, donde el objetivo es el desarrollo local sustentable y los instrumentos se acercan más a un enfoque espacialista.

Finalmente, la instrumentación de las políticas parece limitada a la voluntad de los ejecutivos locales, ya que la falta de una instrumentación clara puede posibilitar el abandono del plan.

Este conjunto de dificultades se acentúa aun más cuando los gobiernos locales disponen de un plazo de 3 o 4 años, no solo para producir la política de ordenamiento territorial, sino para conseguir resultados visibles. Este tiempo de gobierno posibilita aun más el reemplazo del planeamiento reglado y consensuado por objetivos más concretos, como la dotación de infraestructura o de vivienda, con total ausencia de coherencia territorial.

Sin embargo, este análisis, que busca identificar problemas, limitaciones y obstáculos en la fase de formulación de las políticas, debe contextualizarse también junto a otro conjunto de factores que condicionan la capacidad de gestión municipal, como la debilidad en la 
Formulación de políticas de ordenamiento territorial en municipios de México y Argentina a comienzos del siglo XXI: dificultades para su construcción

formación de recursos humanos, la limitada capacidad de gestión por el escaso desarrollo de las estructuras administrativas municipales, los bajos niveles de ingresos, la continuidad del excesivo centralismo, entre otros aspectos.

Si bien este marco es poco auspicioso para que los municipios puedan alcanzar mayor preponderancia en cuanto a su rol en la formulación de políticas, y los resultados conseguidos hasta el momento en relación con las políticas de ordenamiento en proceso de implementación son los presentados, puede entenderse que nos encontramos en un nuevo contexto que conduce a instancias de mayor valoración del territorio, y consecuentemente de las políticas de desarrollo y ordenamiento.

\section{BIBLIOGRAFÍA}

AGUILAR VILLANUEVA, Luis (1ra. Edición, 1993; 2000, 3ra. Edición): Problemas Públicos y Agenda de Gobierno. Colección Antologías de Política Pública III. Editorial Miguel Ángel Porrúa.

BOISIER, Sergio (2003). "Post-scriptum sobre desarrollo regional: Modelos reales y modelos mentales". En EURE Número 72, Mayo, año/vol. XXIX. Pontificia Universidad Católica de Chile. Santiago de Chile.

CHABALGOITY, Miguel (2004). "La Ordenación del Territorio: Aportes a un Debate Nacional". Ponencia expuesta en el $V$ Coloquio sobre Transformaciones Territoriales: Nuevas Visiones en el Inicio del Siglo XXI, La Plata.

DIRECCIÓN NACIONAL DE ORDENAMIENTO TERRITORIAL (DINOT), Ministerio de Vivienda, Ordenamiento Territorial y Medio Ambiente, República Oriental del Uruguay (1996). "Agenda de las Políticas Territoriales en el Cambio De Siglo", Volumen II del Informe Intermedio.

EVANS, Peter (2003). "Hybridity as an Administrative Strategy: Combining Bureaucratic Capacity with Market Signals and Deliberative Democracy", Revista del CLAD Reforma y Democracia. No. 25. Caracas. (Citado en Sciara, et al., 2007).

MADOERY, Oscar (2008) Otro Desarrollo: El cambio desde las ciudades y regiones. Ed. UNSAM edita. Buenos Aires, Argentina.

ORTIZ, Ana Patricia; MASSIRIS, Ángel (1993) Bases para el ordenamiento del Pacífico colombiano, en: El Pacífico colombiano (Santafé de Bogotá, Fondo Eléctrico Nacional -FEN-, Tomo II) 847-872. Citado en Ordenamiento Territorial y Procesos de Construcción Regional de Massiris Cabeza, Ángel. 
OSBORNE, D. and T. GAEBLER (1992). "Reinventing Government: How the Entrepreneurial Spirit Is Transforming the Public Sector". Reading, MA: Addison-Wesley. (Citado en ScIARA, et al., 2007.)

PEÑÍN, Alberto (2006) Hacia un nuevo planeamiento general: Urbanismo y Crisis. Editorial Tribuna De La Construcción. Valencia, España.

RÍOS, L.; ROCCA M. J. y BONO N. (2009). "Desarrollo Local y Ordenamiento Territorial en la Provincia de Buenos Aires". Artículo publicado em GEOGRAFAR Vol $3 N^{o}$ 2. Revista do Programa de Pos Graduacao em Geografia, Págs. 59-85. (Jul-Dic. 2008). Curitiba, Brasil.

SCIARA, A.; RAPOSO, I,; GORBÁN, P.; CAFARELL S. (2007). "Las políticas públicas y la formación de agendas: Las infraestructuras estratégicas desde una mirada regional", ponencia presentada en el $4^{\circ}$ Congreso Argentino de Administración Pública "Sociedad, Gobierno y Administración”, Subsecretaría de la Gestión Pública, la Asociación Argentina de Estudios de Administración Pública y la Asociación de Administradores Gubernamentales. Buenos Aires, Argentina.

SERRANO, Antonio (2004). "La Ordenación del Territorio y el Urbanismo desde la Sostenibilidad". $1^{\circ}$ Foro de Urbanismo 2003, CSCAE, Palma de Mallorca. Citado en Hacia un nuevo planeamiento general: Urbanismo y Crisis de Peñín Alberto.

UNLP - Facultad de Arquitectura y Urbanismo (2009). "Posibles implicancias de la División Territorial del Partido de Chascomús. Dimensión Urbana y Territorial”. Informe final.

\section{DOCUMENTOS PÚBLICOS}

\section{Municipio de Tequisquiapan}

Presupuestos de Recursos, 2002, 2005 y 2008. Publicados en el periódico oficial del Estado Libre y Soberano de Querétaro Arteaga del 14/1/2002, 31/12/2004, y 30/12/2008. Disponibles en: http://www.queretaro.gob.mx/servicios/LaSombradeArteaga

Manual General de Organización del Municipio de Tequisquiapan, Querétaro. Publicado en el periódico oficial del Estado Libre y Soberano de Querétaro Arteaga del 27/03/2009. http://www.queretaro.gob.mx/servicios/LaSombradeArteaga/2009/20090321.html Plan de Desarrollo Urbano del Centro de Población de Tequisquiapan. 2005.

Municipalidad de Chascomús

Presupuestos de Recursos, 2007, 2008 y 2009. Programa RAFAM. Disponibles em: www. chas.mun.gba.gov.ar/Docs

Lineamientos Estratégicos de Desarrollo Local en Chascomús. Documento Base de Diagnóstico. Abril de 2005.nóstico. Abril de 2005. 